\title{
PERFIL ISOENZIMÁTICO DE MAÍCES NATIVOS DEL ISTMO DE TEHUANTEPEC, OAXACA, MÉXICO. II. VARIACIÓN DENTRO DE GRUPOS
}

\author{
ISOZYMATIC PROFILE OF NATIVE MAIZES FROM THE TEHUANTEPEC ISTHMUS, OAXACA, \\ MÉXICO. II. VARIATION WITHIN GROUPS
}

\author{
Gustavo López Romero, Amalio Santacruz Varela*, Abel Muñoz Orozco, Fernando Castillo González, \\ Leobigildo Córdova Téllez y Humberto Vaquera Huerta ${ }^{1}$
}

Colegio de Postgraduados, Campus Montecillo. km 36.5 Carretera México-Texcoco. 56230, Montecillo, Texcoco, Edo. de México. Tel. 01-595-9520200 Ext. 1570, Fax 01-595-9520262

*Autor para correspondencia (asvarela@colpos.mx)

\section{RESUMEN}

A partir de un estudio de 120 poblaciones de maíz (Zea mays $\mathbf{L}$.) nativas del Istmo de Tehuantepec, Oaxaca, México, previamente caracterizadas morfológicamente, se seleccionaron 40 poblaciones representativas para analizar su diversidad genética mediante polimorfismos de 19 loci de isoenzimas. Las 40 poblaciones correspondieron a dos grupos; el primero, integrado por 21 poblaciones de maíz de la raza Zapalote Chico; y el segundo, por 19 poblaciones conocidas localmente como Maíz Grande. Ambos grupos fueron a su vez subdivididos en tres subgrupos cada uno identificados como precoz, intermedio y tardío. Los estimadores de la diversidad genética mostraron valores altos para el grupo de poblaciones de Maíz Grande, con mayor número de alelos por locus, polimorfismo y heterocigosidad esperada, así como una diferenciación genética entre sus poblaciones mayor que la de Zapalote Chico. En Zapalote Chico se observó que el subgrupo tardío presentó los valores más bajos para los estimadores de diversidad genética y de diferenciación genética entre sus poblaciones, mientras que en el subgrupo intermedio tales valores fueron altos. Entre los tres subgrupos de Maíz Grande el subgrupo precoz presentó los valores más bajos diversidad y de diferenciación genética entre sus poblaciones, mientras que el tardío tuvo un mayor número de alelos por locus, y el intermedio presentó los valores más altos para polimorfismo, heterocigosidad esperada y una mayor diferenciación genética entre sus poblaciones.

Palabras clave: Zea mays, Zapalote Chico, Maíz Grande, diversidad genética, polimorfismo.

\section{SUMMARY}

Forty representative maize (Zea mays L.) populations were selected on the basis of information derived from a former study on morphological characterization out of 120 populations native to the Tehuantepec Isthmus, Oaxaca, México, in order to analyze their genetic diversity through polymorphisms of 19 isozyme loci. Native populations corresponded to two groups; the first one was assembled by 21 populations belonging to the Zapalote Chico race, while the second one included 19 populations locally known as Maíz Grande. Both groups were in turn subdivided into three subgroups, identified as early, intermediate, and late maturing. Estimators of genetic diversity showed high values for the group of Maíz Grande populations, with a larger number of alleles per locus, polymorphism and expected heterocigosity, as well as genetic differentiation among their populations larger than Zapalote Chico. Regarding Zapalote Chico, its late subgroup showed the lowest values for the estimators of genetic diversity and genetic differentiation among populations, while the intermediate subgroup had the highest values. In the Maíz Grande subgroups, the early subgroup had the lowest values of genetic diversity and genetic differentiation among its populations, and the late subgroup had a larger number of alleles per locus; nevertheless, the intermediate subgroup had the highest values for polymorphism, expected heterocigosity and a higher genetic differentiation value among its populations.

Index words: Zea mays, Zapalote Chico, Maíz Grande, genetic diversity, polymorphism, isozymes

\section{INTRODUCCIÓN}

La diversidad genética de una especie representa la variación heredable dentro y entre sus poblaciones, y en las especies cultivadas ésta tiene gran trascendencia pues es sobre la cual operan los procesos de selección que aplican los agricultores y fitomejoradores; por ello es necesario caracterizarla a fin de plantear esquemas más eficientes para su aprovechamiento y conservación, mediante las herramientas de la biosistemática. En las razas mexicanas de maíz (Zea mays L.), la caracterización morfológica y genética es un proceso inconcluso, pues sólo se han hecho algunos trabajos para estudiar la variabilidad existente entre razas, que incluyeron un alto número de razas con pocas poblaciones representativas de cada una, lo que ha permitido visualizar un panorama general y caracterizar la variación genética entre razas, pero se ha explorado insuficientemente la variación dentro de razas 
mediante variables morfológicas y polimorfismo de isoenzimas (Doebley et al., 1985; Sánchez et al., 2000). De los estudios de caracterización dentro de razas pueden mencionarse solamente los siguientes: el realizado en la raza Cónico (Silva, 1992; Com. Pers.) ${ }^{1}$, la descripción de diversidad de la raza Chalqueño y sus variantes (HerreraCabrera et al., 2004), y la caracterización morfológica e isoenzimática de la raza Purépecha (Mijangos-Cortés et al., 2007).

El maíz más representativo del Istmo de Tehuantepec es la raza Zapalote Chico, en la que los trabajos de mejoramiento y caracterización también son escasos. Al respecto, Ramírez et al. (1988) realizaron selección familial y concluyeron que las poblaciones de la raza Zapalote Chico tenían estrecha variabilidad genética; posteriormente, 87 poblaciones del Istmo de Tehuantepec fueron caracterizadas y algunas sobresalientes fueron seleccionadas por características agronómicas y rendimiento para iniciar un programa de mejoramiento genético participativo (Aragón et al., 2004; Com. Pers.) ${ }^{2}$. Recientemente, López et al. (2005) caracterizaron morfológicamente 120 poblaciones del Istmo de Tehuantepec, y las clasificaron en dos grandes grupos con tres subgrupos cada uno, con base en su precocidad.

En el presente trabajo se seleccionaron 40 poblaciones pertenecientes a los seis subgrupos del patrón de variación descrito por López et al. (2005), con los objetivos de cuantificar la diversidad genética de tales subgrupos y su grado de diferenciación, a través del contenido alélico de un conjunto de loci de isoenzimas, y de examinar las relaciones de afinidad entre dichos grupos a partir de distancias genéticas generadas con alelos isoenzimáticos. Tal información constituye una valiosa herramienta para definir la estructura genética de los maíces de la región, porque aporta información detallada sobre la diversidad genética disponible para los programas de fitomejoramiento y, en su caso, para el diseño de mejores estrategias de conservación de la misma.

\section{MATERIALES Y MÉTODOS}

\section{Material genético}

A partir de la caracterización morfológica de 120 poblaciones de maíz del Istmo de Tehuantepec reportada

\footnotetext{
${ }^{1}$ Silva Cifuentes, E G (1992) Estudio agronómico y taxonómico de colecciones de la raza de maíz Cónico, su colección central y perspectivas de uso en mejoramiento genético. Tesis de Maestría en Ciencias. Colegio de Postgraduados. Montecillo, Texcoco, Edo. de México. 116 p.

${ }^{2}$ Aragón C F, S Taba, J M Cabrera T, V Chávez (2004) Diversidad genética del maíz Zapalote Chico. In: Memoria del XX Congreso Nacional de Fitogenética. Resúmenes. Sociedad Mexicana de Fitogenética. Chapingo, México. p. 288.
}

por López et al. (2005), se seleccionaron 40 poblaciones representativas del patrón morfológico ahí descrito, las cuales incluyeron 21 poblaciones del grupo de Zapalote Chico; de ellas, siete son del subgrupo precoz, nueve del intermedio y cinco del tardío, cuyos datos de origen y la constitución de subgrupos se indican en el Cuadro 1; y 19 poblaciones del grupo de Maíz Grande, de las cuales cinco corresponden al subgrupo precoz, cuatro al intermedio y 10 al tardío (Cuadro 2). Adicionalmente, se evaluaron 10 poblaciones representativas de otras razas que pudieran estar relacionadas con el maíz del Istmo de Tehuantepec (Cuadro 3). La ubicación geográfica de las poblacions del Istmo de Tehuantepec se señala en la Figura 1.

\section{Evaluación isoenzimática}

Se analizaron 19 loci de las enzimas fosfatasa ácida (Acp1 y Acp4), alcohol deshidrogenasa (Adh1), catalasa (Cat3), esterasa (E8), $\beta$-glucosidasa (Glu1), glutamato oxaloacetato transaminasa (Got1, Got2 y Got3), isocitrato deshidrogenasa (Idh1, Idh2), malato deshidrogenasa (Mdh1, Mdh2, Mdh3, Mdh4 y Mdh5), 6-fosfogluconato deshidrogenasa (Pgd1 y $P g d 2)$ y fosfato isomerasa (Phil), los que están distribuidos en ocho de los 10 cromosomas del maíz (Stuber et al., 1988). Las enzimas se extrajeron a partir de coleoptilos de 10 plántulas de $6 \mathrm{~d}$ de edad en cada población, y de individuos de las líneas testigo B73, Mo17, Tx303 y Mo24W, todas de alelos conocidos. Posteriormente, las enzimas fueron separadas mediante electroforesis en geles de almidón. Después de la tinción, los zimogramas fueron fotografiados para su lectura, y como referencia se tomó el desplazamiento de alelos conocidos de las líneas testigo y se consideraron los patrones de bandeo, todo ello con base en los protocolos de Stuber et al. (1988).

\section{Análisis de la diversidad genética}

A partir de los datos generados de la lectura de los zimogramas, se calcularon las frecuencias génicas por subgrupo de maíces nativos y para el grupo de poblaciones representativas; se determinó también el número de alelos por locus, el porcentaje de loci polimórficos con un criterio de $95 \%$, la heterocigosidad observada y esperada. El grado de diferenciación genética entre poblaciones (Gst) se estimó con el método de Nei (1973) mediante la fórmula $\mathrm{GsT}_{\mathrm{T}}=1-\left(\mathrm{H}_{\mathrm{s}} / \mathrm{H}_{\mathrm{t}}\right)$, donde $\mathrm{H}_{\mathrm{s}}$ es la heterocigosidad esperada promedio por población y $\mathrm{H}_{\mathrm{t}}$ es la heterocigosidad esperada promedio por grupo. Los parámetros anteriores se obtuvieron con los programas POPGENE Versión 1.31 (Yeh et al., 1999) y TFPGA (Miller, 1997). 
Cuadro 1. Procedencia de poblaciones de maíz Zapalote Chico de los subgrupos precoz (A), intermedio (B) y tardío (C) evaluadas para polimorfismo de isoenzimas.

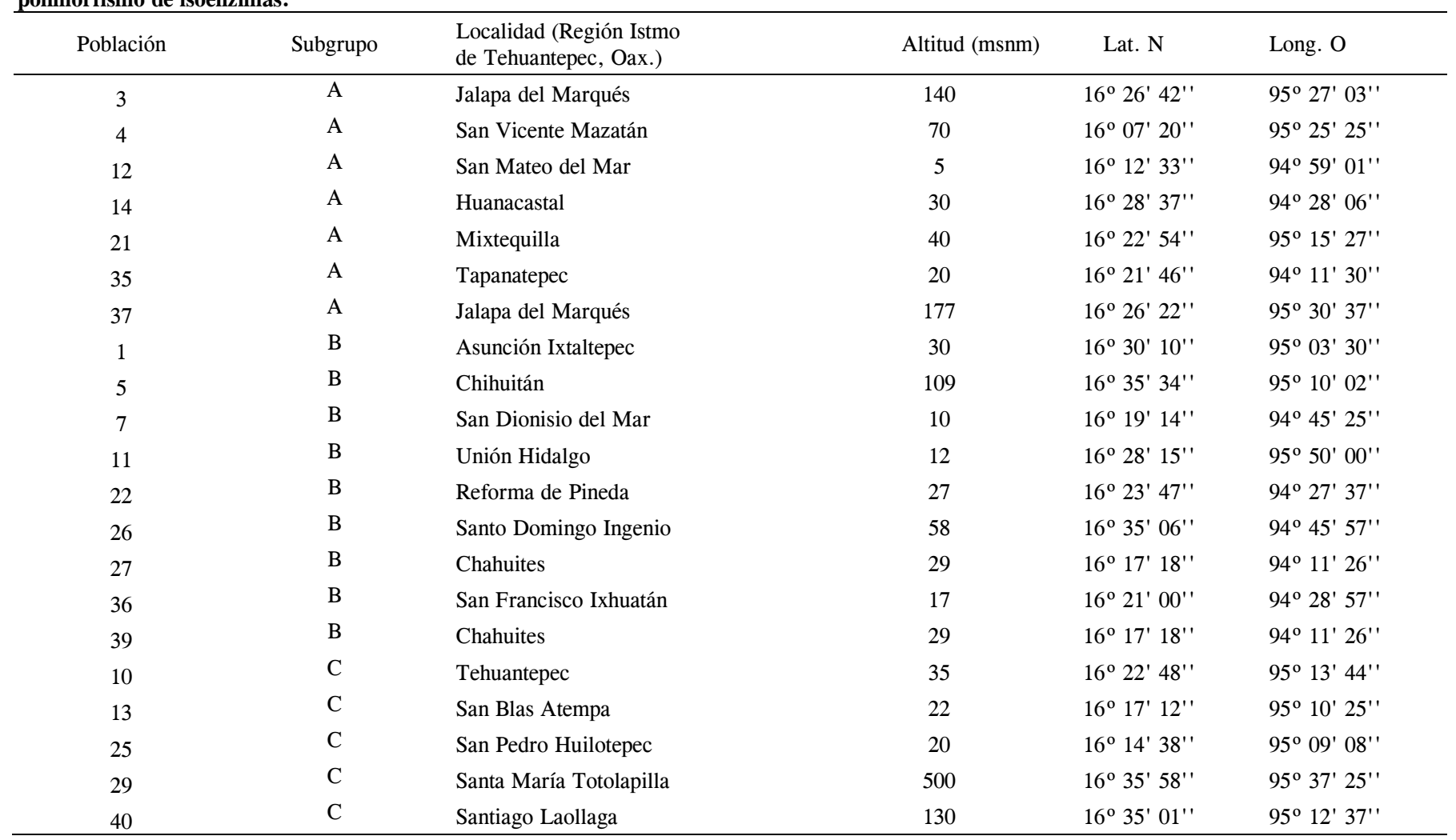

Cuadro 2. Procedencia de poblaciones de Maíz Grande de los subgrupos precoz (D), intermedio (E) y tardío (F), evaluadas para polimorfismo de isoenzimas.

\begin{tabular}{|c|c|c|c|c|c|}
\hline Población & Subgrupo & $\begin{array}{l}\text { Localidad (Región Istmo de Tehuantepec, } \\
\text { Oax.) }\end{array}$ & Altitud (msnm) & Lat. $\mathrm{N}$ & Long. O \\
\hline 6 & $\mathrm{D}$ & Santo Domingo Petapa & 237 & $16^{\circ} 49^{\prime} 17^{\prime \prime}$ & $95^{\circ} 08^{\prime} 20^{\prime \prime}$ \\
\hline 8 & $\mathrm{D}$ & Santo Domingo Petapa & 237 & $16^{\circ} 49^{\prime} 17^{\prime \prime}$ & $95^{\circ} 08^{\prime} 20^{\prime \prime}$ \\
\hline 9 & $\mathrm{D}$ & Santo Domingo Petapa & 237 & $16^{\circ} 49^{\prime} 17^{\prime \prime}$ & $95^{\circ} 08^{\prime} 20^{\prime \prime}$ \\
\hline 17 & $\mathrm{D}$ & Santa María Guienagati & 350 & $16^{\circ} 44^{\prime} 15^{\prime \prime}$ & $95^{\circ} 19^{\prime} 03^{\prime \prime}$ \\
\hline 34 & $\mathrm{D}$ & Santa María Guienagati & 350 & $16^{\circ} 44^{\prime} 15^{\prime \prime}$ & $95^{\circ} 21^{\prime} 14^{\prime \prime}$ \\
\hline 31 & $\mathrm{E}$ & Guevea de Humboldt & 500 & $16^{\circ} 38^{\prime} 49^{\prime \prime}$ & $95^{\circ} 30^{\prime} 33^{\prime \prime}$ \\
\hline 32 & $\mathrm{E}$ & San Juan Guichicovi & 275 & $16^{\circ} 57^{\prime} 30^{\prime \prime}$ & $95^{\circ} 05^{\prime} 30^{\prime \prime}$ \\
\hline 38 & $\mathrm{E}$ & Santo Domingo Ingenio & 58 & $16^{\circ} 35^{\prime} 06^{\prime \prime}$ & $94^{\circ} 45^{\prime} 57^{\prime \prime}$ \\
\hline 2 & $\mathrm{~F}$ & San Pedro Huamelula & 77 & $16^{\circ} 01^{\prime} 30^{\prime \prime}$ & $95^{\circ} 39^{\prime} 57^{\prime \prime}$ \\
\hline 15 & $\mathrm{~F}$ & San Pedro Huamelula & 77 & $16^{\circ} 01^{\prime} 30^{\prime \prime}$ & $95^{\circ} 39^{\prime} 57^{\prime \prime}$ \\
\hline 23 & $\mathrm{~F}$ & Zanatepec & 89 & $16^{\circ} 28^{\prime} 35^{\prime \prime}$ & $94^{\circ} 21^{\prime} 7^{\prime \prime}$ \\
\hline 24 & $\mathrm{~F}$ & San Miguel Tenango & 1550 & $16^{\circ} 16^{\prime} 00^{\prime \prime}$ & $95^{\circ} 36^{\prime} 00^{\prime \prime}$ \\
\hline 28 & $\mathrm{~F}$ & Matías Romero & 180 & $16^{\circ} 52^{\prime} 27^{\prime \prime}$ & $95^{\circ} 02^{\prime} 22^{\prime \prime}$ \\
\hline 30 & $\mathrm{~F}$ & Santa María Totolapilla & 500 & $16^{\circ} 35^{\prime} 58^{\prime \prime}$ & $95^{\circ} 37^{\prime} 25^{\prime \prime}$ \\
\hline 33 & $\mathrm{~F}$ & Santa María Guienagati & 350 & $16^{\circ} 44^{\prime} 15^{\prime \prime}$ & $95^{\circ} 21^{\prime} 14^{\prime \prime}$ \\
\hline
\end{tabular}


Cuadro 3. Poblaciones representativas de otras razas cercanas evaluadas para polimorfismo de isoenzimas.

\begin{tabular}{llll}
\hline Población & Raza representada & Banco donador & Distribución geográfica de la raza \\
\hline LADA-434 & Nal-Tel & UACH $^{\dagger}$ & Península de Yucatán, Chiapas, Oaxaca \\
CHIS-224 & Zapalote Grande & CIMMYT $^{\dagger \dagger}$ & Chiapas, Guerrero y Oaxaca \\
CHIS-578 & Clavillo & CIMMYT & Campeche y Chiapas \\
GUER-96 & Vandeño & CIMMYT & Chiapas, Guerrero y Oaxaca \\
OAXA-54 & Zapalote Chico & CIMMYT & Restringida al Istmo de Tehuantepec, Oax. \\
OAXA-244 & Olotillo & CIMMYT & Chiapas, Oaxaca, San Luis Potosí \\
YUCA-108 & Nal-Tel & CIMMYT & Península de Yucatán, Chiapas, Oaxaca \\
YUCA-117 & Dzit-Bacal & CIMMYT & Península de Yucatán, Chiapas, Veracruz \\
VERA-39 & Tuxpeño & CIMMYT & Llanura del Golfo, Chis., Oax., Ver. \\
VERA-168 & Dzit-Bacal & CIMMYT & Península de Yucatán, Chiapas, Veracruz \\
\hline
\end{tabular}

${ }^{\dagger}$ Universidad Autónoma Chapingo, ${ }^{\dagger \dagger}$ Centro Internacional de Mejoramiento de Maíz y Trigo.

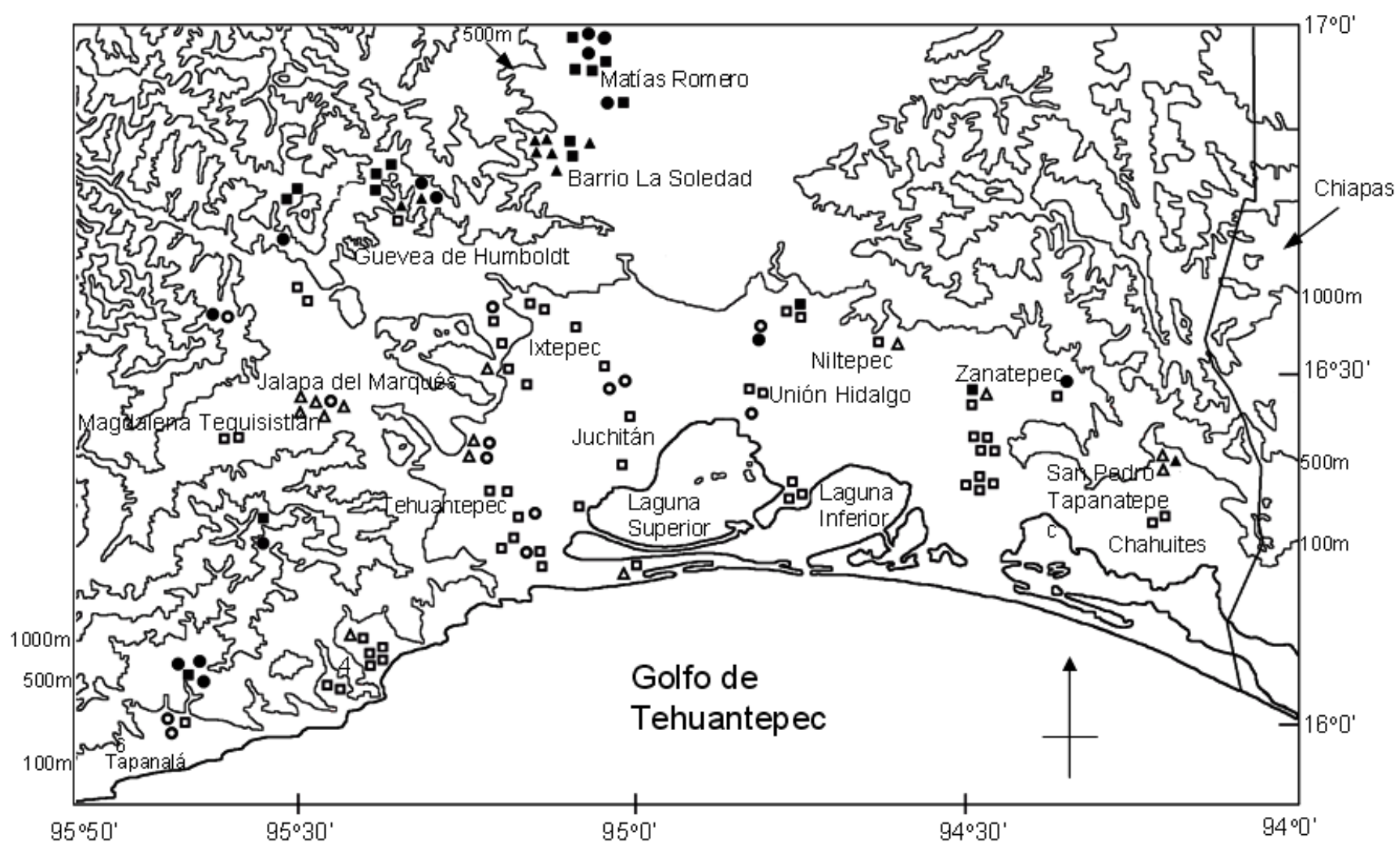

Las líneas de trazo irregular indican curvas de nivel, con su altitud indicada en los márgenes.

Figura 1. Sitios de procedencia de las poblaciones nativas de Zapalote Chico de los subgrupos precoz ( $\Delta$ ), intermedio ( $\square)$ y tardío ( $\circ$ ); y de Maíz Grande de los subgrupos precoz ( $\Delta$ ), intermedio (ø) y tardío (•).

\section{Análisis de agrupamiento}

Con los promedios de las frecuencias génicas para los seis subgrupos y de cada una de las 10 poblaciones representativas, se generó una matriz de distancias genéticas modificadas de Rogers, con el paquete NTSYS- pc (Rohlf, 1993). Con esta matriz se generó un filograma con el método de agrupamiento de vecinos (NeighborJoining) (Saitou y Nei, 1987) mediante el programa Genetic Data Analysis (GDA), versión 1.0 (Lewis y Zaykin, 2001), el cual se visualizó mediante el programa TREEVIEW versión 1.6.1 (Page, 1996). 


\section{RESULTADOS Y DISCUSIÓN}

\section{Frecuencias génicas}

Las frecuencias génicas para 52 alelos observados en los 19 loci se promediaron para cada grupo y subgrupo (Cuadros 4 y 5). Al comparar las frecuencias génicas entre los grupos de Zapalote Chico y Maíz Grande, se observan frecuencias génicas contrastantes para tres loci, Acp1, Glu1 y $P g d 1$, donde el grupo de Zapalote Chico presentó frecuencias altas para los alelos Acp1-2, Glu1-6 (Cuadro 4) y Pgd1-3 (Cuadro 5). En el estudio realizado por Llauradó et al. (1993), al comparar dos grupos de poblaciones de maíz del norte de España, observaron que las frecuencias génicas de los loci Enpl y Mdh3 eran las que más contrastaban entre ambos grupos.

Cuadro 4. Frecuencias génicas de 26 alelos registrados en 8 loci de isoenzimas, promediadas por grupos de poblaciones de acuerdo al análisis de agrupamiento de los caracteres morfológicos.

\begin{tabular}{|c|c|c|c|c|c|c|c|c|c|c|}
\hline \multirow[b]{2}{*}{ Locus-alelo } & \multicolumn{4}{|c|}{ Zapalote Chico } & \multicolumn{4}{|c|}{ Maíz Grande } & \multirow{2}{*}{$\begin{array}{c}\begin{array}{c}\text { Razas } \\
\text { cercanas }\end{array} \\
\text { Media }\end{array}$} & \multirow{2}{*}{$\begin{array}{l}\text { Media } \\
\text { Globa }\end{array}$} \\
\hline & Precoz & Interm. & Tardío & Media & Precoz & Interm. & Tardío & Media & & \\
\hline Acp1-3 & 0.000 & 0.000 & 0.000 & 0.000 & 0.000 & 0.000 & 0.000 & 0.000 & 0.040 & 0.008 \\
\hline Acpl-4 & 0.486 & 0.472 & 0.560 & 0.498 & 0.460 & 0.613 & 0.555 & 0.542 & 0.490 & 0.513 \\
\hline Acp 4-3 & 0.186 & 0.183 & 0.290 & 0.210 & 0.260 & 0.238 & 0.215 & 0.232 & 0.240 & 0.224 \\
\hline Acp 4-4 & 0.450 & 0.500 & 0.500 & 0.483 & 0.550 & 0.513 & 0.535 & 0.534 & 0.420 & 0.490 \\
\hline Acp 4-5 & 0.364 & 0.311 & 0.210 & 0.305 & 0.140 & 0.238 & 0.210 & 0.197 & 0.340 & 0.271 \\
\hline Acp 4-6 & 0.000 & 0.006 & 0.000 & 0.002 & 0.050 & 0.000 & 0.040 & 0.034 & 0.000 & 0.014 \\
\hline Cat3-9 & 1.000 & 0.989 & 1.000 & 0.995 & 1.000 & 1.000 & 0.995 & 0.997 & 0.990 & 0.995 \\
\hline Cat3-12 & 0.000 & 0.011 & 0.000 & 0.005 & 0.000 & 0.000 & 0.005 & 0.003 & 0.000 & 0.003 \\
\hline Est8-4 & 0.914 & 0.961 & 0.900 & 0.931 & 0.910 & 0.900 & 0.960 & 0.934 & 1.000 & 0.946 \\
\hline Est8-5 & 0.029 & 0.000 & 0.020 & 0.014 & 0.060 & 0.075 & 0.010 & 0.037 & 0.000 & 0.020 \\
\hline Est8-6 & 0.021 & 0.011 & 0.000 & 0.012 & 0.000 & 0.000 & 0.020 & 0.011 & 0.000 & 0.009 \\
\hline Est8-8 & 0.036 & 0.028 & 0.080 & 0.043 & 0.030 & 0.025 & 0.010 & 0.018 & 0.000 & 0.025 \\
\hline Glu1-n & 0.014 & 0.022 & 0.000 & 0.014 & 0.060 & 0.150 & 0.000 & 0.047 & 0.020 & 0.028 \\
\hline Glu1-1 & 0.014 & 0.000 & 0.000 & 0.005 & 0.010 & 0.000 & 0.000 & 0.003 & 0.000 & 0.003 \\
\hline Got1-4 & 0.886 & 0.906 & 0.920 & 0.902 & 0.820 & 0.788 & 0.945 & 0.879 & 0.860 & 0.885 \\
\hline Got1-6 & 0.114 & 0.094 & 0.080 & 0.098 & 0.180 & 0.200 & 0.045 & 0.113 & 0.140 & 0.112 \\
\hline
\end{tabular}


Cuadro 5. Frecuencias génicas de 26 alelos registrados de 11 loci de isoenzimas promediadas por grupos de poblaciones de acuerdo al análisis de agrupamiento de los caracteres morfológicos.

\begin{tabular}{|c|c|c|c|c|c|c|c|c|c|c|}
\hline \multirow[b]{2}{*}{ Locus-alelo } & \multicolumn{4}{|c|}{ Zapalote Chico } & \multicolumn{4}{|c|}{ Maíz Grande } & \multirow{2}{*}{$\begin{array}{c}\begin{array}{c}\text { Razas } \\
\text { cercanas }\end{array} \\
\text { Media }\end{array}$} & \multirow{2}{*}{$\begin{array}{l}\text { Media } \\
\text { Global }\end{array}$} \\
\hline & Precoz & Interm. & Tardío & Media & Precoz & Interm. & Tardío & Media & & \\
\hline Got2-4 & 1.000 & 1.000 & 1.000 & 1.000 & 1.000 & 1.000 & 1.000 & 1.000 & 1.000 & 1.000 \\
\hline Got3-4 & 1.000 & 1.000 & 1.000 & 1.000 & 1.000 & 1.000 & 1.000 & 1.000 & 1.000 & 1.000 \\
\hline Idh1-2 & 0.000 & 0.006 & 0.000 & 0.002 & 0.000 & 0.138 & 0.005 & 0.032 & 0.045 & 0.022 \\
\hline$I d h 1-4$ & 1.000 & 0.983 & 1.000 & 0.993 & 1.000 & 0.838 & 0.995 & 0.963 & 0.945 & 0.972 \\
\hline Idh1-6 & 0.000 & 0.011 & 0.000 & 0.005 & 0.000 & 0.025 & 0.000 & 0.005 & 0.010 & 0.006 \\
\hline Idh2-4 & 0.793 & 0.828 & 0.860 & 0.824 & 0.780 & 0.700 & 0.730 & 0.737 & 0.825 & 0.791 \\
\hline $\operatorname{Idh} 2-6$ & 0.207 & 0.172 & 0.140 & 0.176 & 0.220 & 0.300 & 0.270 & 0.263 & 0.175 & 0.209 \\
\hline$M d h 1-1$ & 0.007 & 0.028 & 0.000 & 0.014 & 0.040 & 0.150 & 0.100 & 0.095 & 0.095 & 0.061 \\
\hline$M d h 1-6$ & 0.979 & 0.972 & 0.980 & 0.976 & 0.960 & 0.850 & 0.900 & 0.905 & 0.905 & 0.935 \\
\hline$M d h 1-9.2$ & 0.014 & 0.000 & 0.020 & 0.010 & 0.000 & 0.000 & 0.000 & 0.000 & 0.000 & 0.004 \\
\hline$M d h 2-3$ & 0.564 & 0.556 & 0.540 & 0.555 & 0.600 & 0.600 & 0.635 & 0.618 & 0.635 & 0.595 \\
\hline$M d h 2-5$ & 0.014 & 0.078 & 0.050 & 0.050 & 0.000 & 0.000 & 0.020 & 0.011 & 0.000 & 0.025 \\
\hline$M d h 2-6$ & 0.421 & 0.367 & 0.410 & 0.395 & 0.400 & 0.400 & 0.345 & 0.371 & 0.365 & 0.380 \\
\hline$M d h 3-16$ & 1.000 & 1.000 & 1.000 & 1.000 & 1.000 & 1.000 & 1.000 & 1.000 & 0.980 & 0.996 \\
\hline$M d h 3-18$ & 0.000 & 0.000 & 0.000 & 0.000 & 0.000 & 0.000 & 0.000 & 0.000 & 0.020 & 0.004 \\
\hline$M d h 4-12$ & 0.993 & 0.983 & 1.000 & 0.991 & 0.980 & 0.963 & 0.980 & 0.976 & 0.905 & 0.968 \\
\hline$M d h 4-14.5$ & 0.007 & 0.017 & 0.000 & 0.010 & 0.020 & 0.038 & 0.020 & 0.024 & 0.095 & 0.032 \\
\hline$M d h 5-12$ & 0.700 & 0.789 & 0.820 & 0.767 & 0.700 & 0.775 & 0.800 & 0.768 & 0.650 & 0.744 \\
\hline$M d h 5-15$ & 0.300 & 0.211 & 0.180 & 0.233 & 0.300 & 0.225 & 0.200 & 0.232 & 0.350 & 0.256 \\
\hline Pgd1-2 & 0.486 & 0.489 & 0.280 & 0.438 & 0.580 & 0.550 & 0.610 & 0.590 & 0.480 & 0.504 \\
\hline Pgd1-3.8 & 0.514 & 0.511 & 0.720 & 0.562 & 0.420 & 0.450 & 0.390 & 0.411 & 0.520 & 0.496 \\
\hline $\operatorname{Pgd} 2-5$ & 1.000 & 1.000 & 1.000 & 1.000 & 1.000 & 1.000 & 1.000 & 1.000 & 1.000 & 1.000 \\
\hline Phil-2 & 0.000 & 0.000 & 0.050 & 0.012 & 0.000 & 0.013 & 0.000 & 0.003 & 0.000 & 0.006 \\
\hline Phil-3 & 0.171 & 0.161 & 0.100 & 0.150 & 0.090 & 0.113 & 0.070 & 0.084 & 0.000 & 0.095 \\
\hline Phil-4 & 0.779 & 0.828 & 0.840 & 0.814 & 0.890 & 0.875 & 0.920 & 0.903 & 0.990 & 0.883 \\
\hline Phil-5 & 0.050 & 0.011 & 0.010 & 0.024 & 0.020 & 0.000 & 0.010 & 0.011 & 0.010 & 0.016 \\
\hline
\end{tabular}

Entre los tres subgrupos de Zapalote Chico, el tardío se distinguió por presentar una frecuencia alta del alelo Acp14, con una alelo exclusivo (Phil-2) y cinco alelos ausentes (Est8-6, Glu1-n, Glu1-7, Mdh1-1, Mdh4-14.5); el intermedio se caracterizó por presentar cuatro alelos exclusivos (Acp4-6, Cat3-12, Idh1-2, Idh1-6) y dos alelos ausentes (Est8-5, Mdh1-9.2); mientras que el subgrupo precoz se distinguió de los otros dos por presentar a Glu11 como alelo exclusivo (Cuadros 4 y 5).
Entre los subgrupos de Maíz Grande, el subgrupo tardío se distinguió de los otros dos por presentar tres alelos exclusivos (Cat3-12, Est8-6 y Mdh2-5) y un alelo ausente (Glu1-n); el intermedio presentó otros tres alelos exclusivos (Acp4-2, Idh1-6 Phi1-2) y dos alelos ausentes (Acp4-6, Phi1-5); y el precoz mostró una frecuencia alta del alelo Acp1-2, dos alelos exclusivos (Glu1-1, Glu1-8) y dos ausentes (Got1-2, Idh1-2) (Cuadros 4 y 5). Es difícil precisar el origen de las diferencias de frecuencias alélicas entre poblaciones; sin embargo, puede afirmarse que la selección no es la fuerza predominante de este fenómeno, 
ya que las isoenzimas son marcadores selectivamente neutros, o muy cercanos a tal condición (Kimura, 1983). Por tanto, es más probable que lo anterior pueda atribuirse a efectos de deriva genética o mutación, fenómenos que tienen un fuerte componente aleatorio.

El porcentaje de alelos con frecuencia por debajo de 0.05 fue de $17 \%$ para las poblaciones de Maíz Grande, y de $12 \%$ para las de Zapalote Chico; entre los seis subgrupos, esta variación sólo fue del orden de 0 a $5 \%$. Valores semejantes a los aquí obtenidos para alelos de baja frecuencia han sido reportados; por ejemplo, Smith (1986) reportó 8 de 56 alelos (14\%) en 49 poblaciones de polinización libre del complejo racial dentado de la faja maicera; Revilla y Tracy (1995) reportaron 13 de 60 alelos (22\%) en poblaciones de maíz dulce. Santacruz-Varela et al. (2004) observaron cinco de 58 alelos (8\%) en maíces palomeros. Un caso extremo es el reportado por Sánchez et al. (2000) para 59 razas de México, para las cuales, de 303 alelos observados 194 alelos (64\%) fueron alelos raros.

Al comparar las frecuencias génicas del grupo de Zapalote Chico y Maíz Grande con respecto a otros estudios para los loci aquí estudiados, se encontró que las 19 poblaciones de Maíz Grande del Istmo de Tehuantepec se caracterizan por tener una frecuencia relativamente alta del alelo Pgd1-2 (Cuadro 5), mientras que en la mayoría de estudios con otros materiales el alelo predominante es el Pgd1-3.8 (Goodman y Stuber, 1983; Smith, 1986; Doebley et al., 1988; Bretting et al., 1990; Sánchez et al., 2000; Revilla y Tracy, 1995; Revilla et al., 1998; Santacruz-Varela et al., 2004). Las poblaciones de Maíz Grande, al igual que 47 poblaciones de España reportadas por Revilla et al. (1998), tuvieron frecuencias altas del alelo Glu1-2, mientras que las de Zapalote Chico y 66 poblaciones de maíz dulce reportadas por Revilla y Tracy (1995) mostraron una frecuencia alta del alelo Glu1-6; otros estudios mostraron frecuencia alta para el alelo Glu17 (Goodman y Stuber, 1983; Smith, 1986; Doebley et al., 1988; Bretting et al., 1990; Sánchez et al., 2000; Santacruz-Varela et al., 2004).

En el locus $A c p 1$ se observó una frecuencia alta para el alelo Acp1-2 en las poblaciones del grupo Zapalote Chico, al igual que en las razas de Bolivia (Goodman y Stuber, 1983), de México (Sánchez et al., 2000), en las de maíz palomero (Santacruz-Varela et al., 2004) y en poblaciones de España (Revilla et al., 1998), mientras que el alelo Acp 1-4 tuvo frecuencia relativamente alta en poblaciones de Maíz Grande, en las razas de Guatemala (Bretting et $a l$., 1990), en maíz dulce (Revilla y Tracy, 1995), y en maíces cristalinos del norte (Doebley et al., 1988).
Los resultados anteriores muestran que los maíces de diferentes regiones han cambiado en diferente medida sus frecuencias génicas, debido posiblemente a uno o varios factores como: deriva genética, mutación, cambios en la presión de selección asociados con desplazamientos a nuevos ambientes y aislamiento reproductivo (Doebley et al., 1988). Tal situación sido documentada para los maíces de Guatemala y Sur de México, donde se detectaron cambios en el patrón isoenzimático asociados con la altitud (Bretting y Goodman, 1990), fenómeno que al parecer también se presenta en los maíces del Istmo de Tehuantepec, Oaxaca, y fue notorio que las poblaciones de Zapalote Chico acumularon alelos que las distinguen de las de Maíz Grande.

\section{Número de alelos}

El Cuadro 6 muestra algunos parámetros de diversidad, con la media y desviación estándar por cada grupo y subgrupos dentro de ellos. Respecto al número de alelos, las poblaciones de Zapalote Chico tuvieron un promedio de 2.42 y las de Maíz Grande tuvieron un promedio de 2.47 alelos por locus, mientras que el grupo de 10 poblaciones de otras razas mostraron 2.05 alelos por locus (Cuadro 6). En los seis subgrupos estudiados se observó variación en el número promedio de alelos, desde 1.8 en el subgrupo tardío de Zapalote Chico hasta 2.2 en el intermedio del mismo grupo. El menor número de alelos en las poblaciones del subgrupo tardío de Zapalote Chico, sugiere una diversidad genética relativamente baja en estas poblaciones, posiblemente relacionada con el ambiente de cultivo ya que estas poblaciones predominan en condiciones de riego, lo que puede redundar en una menor variación genética, la cual debe ser alta para sobrevivir en ambientes de mayor riesgo como en el caso de las poblaciones de temporal o secano. Aunado a ello, los ciclos de selección recurrente, la intensidad de selección y los criterios de selección de los productores, pudieron influir en la obtención de poblaciones con un alta proporción de alelos fijados (Sánchez et al., 2000).

En comparación con otros estudios que consideran el mismo conjunto de marcadores usados en este trabajo, Bretting et al. (1990) y Sánchez et al. (2000) encontraron valores de 6.4 y 9.3 alelos por locus, respectivamente, muy superiores a los obtenidos en este trabajo para las poblaciones de Zapalote Chico y Maíz Grande. El locus con el mayor número de alelos en este trabajo fue GluI con un total de 6 alelos, lo que está en concordancia con los altos valores de polimorfismo encontrados en este locus en otros estudios (Goodman y Stuber, 1983; Bretting et al., 1990; Sánchez et al., 2000), y es de destacarse que en este último se usó una gama de materiales muy diversos, con poblaciones de 59 razas. 
Cuadro 6. Parámetros de diversidad genética de 19 loci de isoenzimas sobre los subgrupos de Zapalote Chico (ZCh) y Maíz Grande (MG) y de poblaciones representativas de razas cercanas.

\begin{tabular}{|c|c|c|c|c|c|c|c|}
\hline \multirow[b]{2}{*}{ Grupo } & \multicolumn{2}{|c|}{ Numero de alelos por locus } & \multicolumn{2}{|c|}{ Heterocigosidad observada } & \multicolumn{2}{|l|}{$\begin{array}{l}\text { Heterocigosidad } \\
\text { esperada } \\
\end{array}$} & \multirow{2}{*}{$\begin{array}{c}\begin{array}{c}\text { Loci } \\
\text { polimórficos (\%) }\end{array} \\
\text { Criterio } 95 \%\end{array}$} \\
\hline & Media & Des. est. & Media & Des. est. & Media & Des. est. & \\
\hline \multicolumn{8}{|c|}{ Zapalote Chico } \\
\hline Precoz & 2.16 & 1.302 & 0.141 & 0.187 & 0.219 & 0.232 & 58 \\
\hline Intermedio & 2.21 & 1.084 & 0.133 & 0.180 & 0.208 & 0.226 & 54 \\
\hline Tardío & 1.89 & 0.937 & 0.132 & 0.185 & 0.195 & 0.218 & 58 \\
\hline Grupal & 2.42 & 1.346 & 0.135 & 0.182 & 0.210 & 0.225 & 58 \\
\hline
\end{tabular}

Maíz Grande

\begin{tabular}{llllllll} 
Precoz & 2.053 & 1.268 & 0.145 & 0.195 & 0.221 & 0.230 & 63 \\
Intermedio & 2.105 & 0.994 & 0.143 & 0.178 & 0.254 & 0.224 & 68 \\
Tardío & 2.158 & 0.958 & 0.130 & 0.181 & 0.205 & 0.225 & $\mathbf{0 . 2 2 2}$ \\
Grupal & $\mathbf{2 . 4 7 4}$ & $\mathbf{1 . 4 2 9}$ & $\mathbf{0 . 1 3 7}$ & $\mathbf{0 . 1 7 9}$ & $\mathbf{0 . 2 2 4}$ & $\mathbf{6 8}$ \\
& & & & & & $\mathbf{0 . 2 2 4}$ & $\mathbf{0 . 2 3 0}$ \\
Razas cercanas & $\mathbf{2 . 0 5 3}$ & $\mathbf{0 . 9 7}$ & $\mathbf{0 . 1 3 5}$ & $\mathbf{0 . 1 8 9}$ & & $\mathbf{7 4}$ \\
\hline
\end{tabular}
Des. est. = Desviación estándar.

\section{Porcentaje de loci polimórficos}

Con respecto al porcentaje de loci polimórficos (criterio de $95 \%$ ), los datos del Cuadro 6 muestran un menor porcentaje de loci polimórficos para las poblaciones de Zapalote Chico (58 \%), y mayor para el grupo de Maíz Grande (68\%). La mayor variabilidad del Maíz Grande puede tener su origen en la existencia de cierta introgresión en este grupo con las razas Vandeño, Tepecintle y Tuxpeño, tal como reportaron López et al. (2005). Entre los seis subgrupos se observó que los valores extremos de polimorfismo se encuentran entre ambos subgrupos intermedios, con $54 \%$ para el de Zapalote Chico y $74 \%$ para el intermedio de Maíz Grande. Al comparar los mismos loci valuados en este estudio, otros autores han reportado un valor mayor en las razas de Guatemala (Bretting et al., 1990) y de Bolivia (Goodman y Stuber, 1983), ambas con $88.9 \%$.

\section{Heterocigosidad}

La heterocigosidad esperada es una función tanto del número de alelos como de distribución de las frecuencias génicas, y representa la proporción de las frecuencias genotípicas en equilibrio de Hardy-Weinberg, correspondientes al genotipo heterocigote después de una supuesta generación de apareamiento aleatorio. En el
Cuadro 6 se observa que la heterocigosidad esperada para el grupo de poblaciones de Zapalote Chico mostró un valor de 0.210, menor que el del grupo de Maíz Grande y de poblaciones representativas de otras razas con 0.224. Entre los subgrupos se observó una mayor variación, desde 0.195 para el subgrupo tardío de Zapalote Chico hasta 0.254 para el intermedio de Maíz Grande. Al comparar la heterocigosidad esperada con la obtenida en otros estudios, con base en el mismo conjunto de isoenzimas, la heterocigosidad esperada total obtenida para Zapalote Chico y Maíz Grande es comparable con los valores obtenidos por varios autores, de 0.234 en razas de Bolivia (Goodman y Stuber, 1983), 0.250 en el complejo racial dentado de la faja maicera en EE.UU. (Smith, 1986), 0.266 en razas de Guatemala (Bretting et al., 1990), 0.206 en maíces dulces de polinización abierta (Revilla y Tracy, 1995), 0.229 en razas de maíz de España (Revilla et al., 1998), 0.274 en razas mexicanas (Sánchez et al., 2000), y 0.271 en maíces palomeros del continente americano (Santacruz-Varela et al., 2001), lo que demuestra que las poblaciones del Istmo de Tehuantepec no necesariamente son menos diversas.

A nivel de loci individuales, se encontró (datos no mostrados) que para Zapalote Chico y Maíz Grande hay diferencias en los valores de heterocigosidad para los loci Glu1, Idh2 y Phil, y en ambos grupos se apreció una baja 
heterocigosidad para los loci Cat3, Idh1 y Mdh4, los cuales correspondieron también con los menores valores de la heterocigosidad esperada en otros trabajos, excepto para el locus Cat 3 , ya que el promedio general es de 0.23 . Pero la baja heterocigosidad en este locus (0.01) sólo es comparable con el valor de 0.09 obtenido para las razas de maíz de Guatemala (Bretting et al., 1990); valores bajos de heterocigosidad del locus Idh1 también fueron observados por Revilla y Tracy (1995) en poblaciones de maíz dulce, y por Smith (1986) en poblaciones de la faja maicera. El locus $A c p 4$ tuvo la heterocigosidad más alta con 0.63 para Zapalote Chico, comparable con la obtenida por Santacruz-Varela et al. (2004) en poblaciones de maíz palomero del nuevo mundo (0.7). El locus Glu1 mostró el segundo valor mayor de heterocigosidad con 0.59 para Maíz Grande, comparable con valores obtenidos en los trabajos arriba citados, excepto para el caso de Revilla y Tracy (1995) en poblaciones de maíz dulce donde se reportó un valor menor, de 0.29. Estos resultados confirman que existe un valor informativo desigual entre los diferentes loci, por lo que no es recomendable excluir de estudios posteriores a los loci que exhiben mayor polimorfismo, como el caso de Glu1, Idh2 y Phil.

\section{Diferenciación genética entre poblaciones}

En el Cuadro 7 se muestran los valores de Gst de los grupos y subgrupos para los 19 loci en estudio. La mayor diferenciación genética relativa fue en las poblaciones representativas de otras razas $(0.235)$, seguidas del grupo de Maíz Grande (0.131) y al final para las poblaciones de Zapalote Chico (0.10), lo cual probablemente se relaciona con el hecho de que al Maíz Grande se le cultiva en ambientes más heterogéneos, de altitud variable y fragmentados por sistemas orográficos, lo que ocasiona reducción de flujo genético entre poblaciones y por ende una mayor diferenciación, mientras que Zapalote Chico se restringe a condiciones más homogéneas en la planicie costera. En los subgrupos de Maíz Grande se aprecia que el intermedio es el que presenta una mayor diferenciación entre sus poblaciones (0.185) y menor en el precoz (0.061), en tanto que para los subgrupos de Zapalote Chico el tardío mostró la mayor diferenciación $(0.100)$ y la menor fue en el precoz (0.079). La mayor diferenciación genética relativa entre las poblaciones de otras razas puede explicarse por su diferente origen y patrimonio genético.

Cuadro 7. Diferenciación genética relativa (Gst) de 19 loci de isoenzimas por grupos y subgrupos derivados de 50 poblaciones de maíz'

\begin{tabular}{|c|c|c|c|c|c|c|c|c|c|c|}
\hline \multirow[b]{2}{*}{ Locus } & \multicolumn{4}{|c|}{ Zapalote Chico } & \multicolumn{4}{|c|}{ Maíz Grande } & \multirow[b]{2}{*}{$\begin{array}{l}\text { Repr. } \\
(\mathrm{n}=10)\end{array}$} & \multirow[b]{2}{*}{$\begin{array}{l}\text { Total } \\
(\mathrm{n}=50)\end{array}$} \\
\hline & $\begin{array}{l}\text { Precoz } \\
(\mathrm{n}=7)\end{array}$ & $\begin{array}{l}\text { Interm. } \\
(\mathrm{n}=9)\end{array}$ & $\begin{array}{l}\text { Tardío } \\
(\mathrm{n}=5)\end{array}$ & $\begin{array}{l}\text { Grupo } \\
(\mathrm{n}=21)\end{array}$ & $\begin{array}{l}\text { Precoz } \\
(\mathrm{n}=5)\end{array}$ & $\begin{array}{l}\text { Interm. } \\
(n=4)\end{array}$ & $\begin{array}{l}\text { Tardío } \\
(\mathrm{n}=10)\end{array}$ & $\begin{array}{l}\text { Grupo } \\
(\mathrm{n}=19)\end{array}$ & & \\
\hline Acp1 & 0.059 & 0.036 & 0.067 & 0.056 & 0.014 & 0.139 & 0.108 & 0.100 & 0.279 & 0.122 \\
\hline Acp 4 & 0.132 & 0.138 & 0.093 & 0.134 & 0.089 & 0.057 & 0.111 & 0.097 & 0.154 & 0.132 \\
\hline Cat3 & $\mathrm{vi}^{H+}$ & 0.091 & vi & 0.098 & vi & vi & 0.040 & 0.038 & 0.091 & 0.090 \\
\hline Est8 & 0.080 & 0.064 & 0.214 & 0.130 & 0.098 & 0.183 & 0.126 & 0.146 & vi & 0.148 \\
\hline Glu1 & 0.109 & 0.087 & 0.211 & 0.124 & 0.067 & 0.210 & 0.089 & 0.137 & 0.222 & 0.189 \\
\hline Got1 & 0.089 & 0.048 & 0.035 & 0.064 & 0.031 & 0.110 & 0.079 & 0.116 & 0.348 & 0.154 \\
\hline Idh1 & vi & 0.071 & vi & 0.078 & vi & 0.395 & 0.040 & 0.432 & 0.356 & 0.371 \\
\hline Idh2 & 0.032 & 0.078 & 0.061 & 0.062 & 0.027 & 0.363 & 0.163 & 0.180 & 0.185 & 0.146 \\
\hline Mdh1 & 0.067 & 0.105 & 0.082 & 0.094 & 0.036 & 0.069 & 0.067 & 0.079 & 0.375 & 0.186 \\
\hline$M d h 2$ & 0.040 & 0.076 & 0.080 & 0.068 & 0.058 & 0.234 & 0.098 & 0.119 & 0.253 & 0.124 \\
\hline$M d h 3$ & vi & vi & vi & vi & vi & vi & vi & vi & 0.184 & 0.200 \\
\hline Mdh4 & 0.044 & 0.068 & vi & 0.068 & 0.031 & 0.048 & 0.056 & 0.049 & 0.288 & 0.216 \\
\hline Mdh5 & 0.095 & 0.139 & 0.092 & 0.124 & 0.038 & 0.097 & 0.163 & 0.119 & 0.152 & 0.139 \\
\hline Pgd1 & 0.051 & 0.155 & 0.028 & 0.122 & 0.122 & 0.293 & 0.180 & 0.191 & 0.271 & 0.194 \\
\hline Phil & 0.090 & 0.094 & 0.145 & 0.110 & 0.062 & 0.064 & 0.051 & 0.061 & 0.091 & 0.126 \\
\hline Gst & 0.079 & 0.098 & 0.100 & 0.100 & 0.061 & 0.185 & 0.118 & 0.131 & 0.235 & 0.15 \\
\hline
\end{tabular}

${ }^{+}$No se incluyen los loci monomórficos Adh1, Got2, Got3 y $P g d 2 ;{ }_{\mathrm{vi}}=$ Valor indeterminado debido a monomorfismo para un locus en subgrupos particulares. Repr. $=$ Poblaciones representativas de otras razas. 
Estos resultados indican que las poblaciones del Istmo de Tehuantepec tienen un nivel moderado de divergencia genética para los subgrupos de Zapalote Chico, y de moderado a alto entre los subgrupos de Maíz Grande, de acuerdo con el criterio de Snyder et al. (1985), quienes consideran que una divergencia genética moderada posee valores de Gst entre 0.05 y 0.15 , y alta entre 0.15 y 0.25 . En otros estudios se obtuvieron valores similares de Gst para los agrupamientos evaluados; por ejemplo, Llauradó et al. (1993) detectaron un valor de 0.128 para 86 poblaciones del norte de España; para 34 razas de México, Doebley et al. (1985) reportaron un valor de 0.276; un valor extremo de Gst de 0.561 lo reportan SantacruzVarela et al. (2004) para el subgrupo de poblaciones de maíz palomero de los Estados Unidos, atribuible a que éstas se reproducen en viveros con aislamiento genético estricto, que las ha diferenciado de manera marcada mediante la fijación de alelos contrastantes entre ellas. El bajo valor de Gst observado en este trabajo para los subgrupos evaluados se puede atribuir a que el área explorada es pequeña, en comparación con otros trabajos donde se han utilizado muestras representativas a nivel país (Doebley et al., 1985; Pflüger y Schlatter, 1996), mientras que las colecciones estudiadas por Llauradó et al. (1993) provienen de la región costera del norte de España, que cubre un área de $110 \mathrm{mil} \mathrm{km}^{2}$; en contraste, en este estudio el área explorada fue de alrededor de $13 \mathrm{mil} \mathrm{km}^{2}$.

La diferenciación genética relativa (Gst) total fue de 0.15 , lo que indica que $85 \%$ de la variación reside dentro de las poblaciones y sólo $15 \%$ entre poblaciones (Cuadro 7). El valor de 0.15 puede dividirse para obtener la variación entre grupos y entre poblaciones dentro de grupos; para ello, se consideraron los seis subgrupos derivados de las poblaciones nativas y el grupo de poblaciones de razas representativas de otras razas. Así, el valor de la variación entre grupos $\left(\mathrm{G}_{\mathrm{rt}}\right)$ fue de 0.02 , y la variación entre poblaciones dentro de grupos $\left(\mathrm{G}_{\mathrm{sr}}\right)$ de 0.13 . Estos valores indican que hay mayor variación entre poblaciones dentro de grupos que entre grupos, a diferencia de lo encontrado en materiales de Argentina, donde la variación entre razas fue de 0.14 , mayor que la variación entre poblaciones dentro de razas, con valor de 0.08 (Pflüger y Schlatter, 1996).

\section{Análisis de agrupamiento}

Las relaciones de afinidad de los seis subgrupos y de las diez poblaciones de razas representativas se determinaron a partir de la matriz de distancias modificadas de Rogers (Wright, 1978) con las frecuencias de los 52 alelos de isoenzimas detectados, mediante un filograma elaborado con el método de agrupamiento de vecinos (Neighbor-Joining) (Saitou y Nei, 1987), el cual se muestra en la Figura 2. En el filograma se observa que el subgrupo precoz de Maíz Grande guarda una mayor similitud genética con los subgrupos de Zapalote Chico. En el mismo sentido, López et al. (2005) indicaron la similitud morfológica de aquel subgrupo con las poblaciones de Zapalote Chico, que corresponde a la cercanía geográfica donde se cultivan ambos tipos de maíz. Dentro del Maíz Grande, la separación de los subgrupos guarda una estrecha relación con la precipitación de la zona de procedencia; así, el subgrupo precoz proviene del área del Barrio La Soledad cuya precipitación promedio es de $965 \mathrm{~mm}$ anuales (SMN, 2009), en contraste con los maíces intermedios que provienen del área de Guevea de Humboldt, y con los tardíos del área de Matías Romero, con precipitaciones anuales de 1239 y $1691 \mathrm{~mm}$ en promedio (SMN, 2009). Dentro de Zapalote Chico el subgrupo tardío se diferenció del precoz e intermedio probablemente debido a que la mayoría de las poblaciones tardías se cultivan bajo condiciones de riego que aprovechan las aguas de los ríos Tequisistlán y Tehuantepec a través de la presa Lic. Benito Juárez, localizada en Jalapa del Marqués.

Las razas usadas en este trabajo corresponden a las del grupo de dentados tropicales reportado por Sánchez et al. (2000), quienes dividieron ese grupo en dos apartados, el primero integrado por razas adaptadas a elevaciones de bajas a medianas $(0-1700 \mathrm{~m})$ y un segundo con razas adaptadas a mayores altitudes. Es notorio que el agrupamiento que contiene a las razas Tuxpeño, Vandeño, Olotillo y Clavillo guarde similitud con el grupo de elevaciones de bajas a medianas de Sánchez et al. (2000), y en la Figura 2 muestra mayor cercanía genética con los materiales de mayor ciclo vegetativo de Maíz Grande; es decir, con los más alejados físicamente de la planicie costera del Pacífico, probablemente por la dificultad que plantean las condiciones restrictivas de la planicie como altas temperaturas, resequedad y prevalencia de fuertes vientos para que el polen foráneo permanezca viable. También cabe destacar la discrepancia entre las muestras de Nal-Tel, DzitBacal y del propio Zapalote Chico provenientes de los bancos de germoplasma de la UACH y del CIMMYT, pues mientras los materiales de un banco se separan de todos los maíces del Istmo, los del otro muestran cierta asociación con los subgrupos intermedio y tardío de Maíz Grande (Figura 2), lo que pone de manifiesto la necesidad de una revisión a fondo de la documentación de los materiales resguardados en los bancos de germoplasma. 


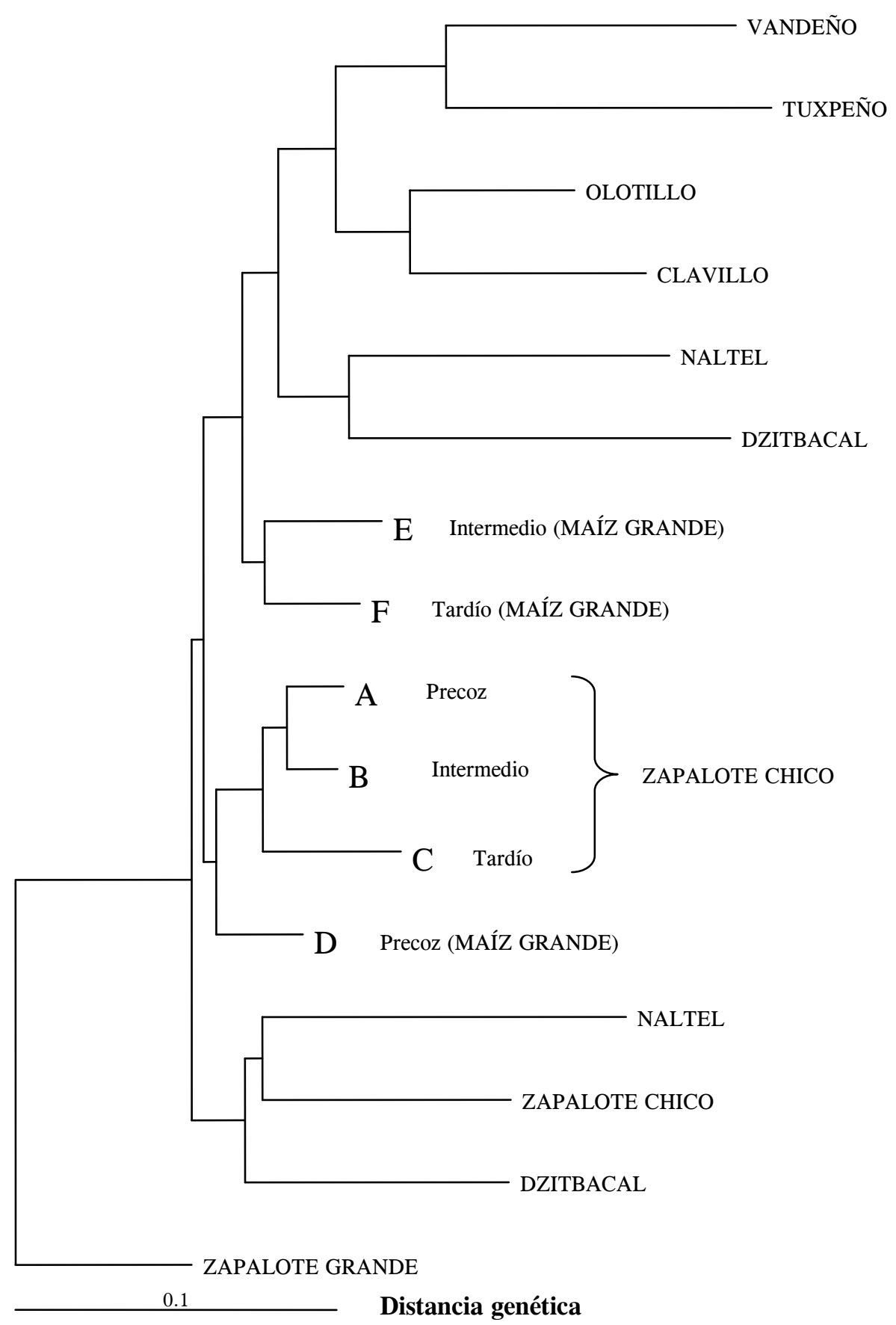

Figura 2. Filograma de los subgrupos precoz (A), intermedio (B) y tardío (C) de Zapalote Chico, y de los subgrupos precoz (D), intermedio (E) y tardío (F) de Maíz Grande, y diez poblaciones representativas de razas del sur de México, mediante la matriz de las distancias modificadas de Rogers derivadas de 52 alelos de isoenzimas con el método de agrupamiento de vecinos (Neighbor-Joining). 


\section{CONCLUSIONES}

Las poblaciones de la raza Zapalote Chico y las denominadas localmente como Maíz Grande, presentaron diferencias isoenzimáticas principalmente en seis loci, de los cuales Glu1, Mdh1 y Pgdl fueron los que más aportaron a la separación de las poblaciones nativas del Istmo de Tehuantepec. Los estimadores de la diversidad genética permitieron establecer que ésta es mayor entre las poblaciones de maíz del grupo Maíz Grande y menor para las poblaciones de Zapalote Chico. Entre los tres subgrupos de Zapalote Chico, el tardío tuvo la más baja diversidad genética y el intermedio la más alta. Entre los subgrupos de Maíz Grande, el precoz tuvo una diversidad genética baja mientras que el intermedio tuvo una diversidad alta entre sus poblaciones. El subgrupo precoz de Maíz Grande resultó genéticamente más similar a las poblaciones de Zapalote Chico.

\section{BIBLIOGRAFÍA}

Bretting P K, M M Goodman, C W Stuber (1990) Isozymatic variation in Guatemalan races of maize. Amer. J. Bot. 77:211-225.

Doebley J F, M M Goodman, C W Stuber (1985) Isozyme variation in races of maize from Mexico. Amer. J. Bot. 72:629-639.

Doebley J F, J D Wendel, J S C Smith, C W Stuber, M M Goodman (1988) The origin of Cornbelt maize: the isozyme evidence. Econ. Bot. 42:120-131.

Goodman M M, C W Stuber (1983) Races of maize. VI. Isozyme variation among races of maize in Bolivia. Maydica 28:169-187.

Herrera-Cabrera B E, F Castillo-González, J J Sánchez-González, J M Hernández-Casillas, R A Ortega-Paczka, M M Goodman (2004) Diversidad del maíz Chalqueño. Agrociencia 38:191-206.

Kimura M (1983) The Neutral Theory of Molecular Evolution. Cambridge University Press. London. 384 p.

Lewis P O, D Zaykin (2001) Genetic Data Analysis: computer program for the analysis of allelic data. Version 1.0 (d16c). Disponible en: http//lewis.eeb.uconn.edu/lewishome/software.html (Junio de 2008).

Llauradó M, J Moreno G, P Arús (1993) Classification of northern Spanish populations of maize by methods of numerical taxonomy. II. Isozyme variation. Maydica 38:249-258

López R G, A Santacruz V, A Muñoz O, F Castillo G, L Córdova T, H Vaquera H (2005) Caracterización morfológica de poblaciones nativas de maíz del Istmo de Tehuantepec, Oaxaca. Interciencia 30:284-290.

Mijangos-Cortés J O, T Corona-Torres, D Espinosa-Victoria, A Muñoz-Orozco, J Romero-Peñaloza, A Santacruz-Varela (2007) Differentiation among maize (Zea mays L.) landraces from the Tarasca Mountain Chain, Michoacan, Mexico and the Chalqueño complex. Genet. Res. Crop Evol. 54:309-325.

Miller M P (1997) Tools for population genetic analyses (TFPGA) 1.3 A Windows program for the analysis of allozyme and molecular population genetic data. Disponible en: http://herb.bio.nau.edu/ miller (Junio de 2008).

Nei M (1973) Analysis of gene diversity in subdivided populations. Proc. Natl. Acad. Sci. USA. 70: 3321-3323.

Page R D M (1996) Treeview: An application to display phylogenetic trees on personal computers. Comput. Appl. Biosci. 12:357-358.

Pflüger L A, A R Schlatter (1996) Isozyme variation in some races of maize from Argentina. Genet. Res. Crop Evol. 43: 357-362.

Ramírez F A, H Ángeles A, J D Molina G (1988) Selección familial de progenies autofecundadas en una variedad de maíz (Zea mays L.) de la raza Zapalote Chico. Agrociencia 74:103-114.

Revilla P, W F Tracy (1995) Isozyme variation and phylogetic relationships among open-pollinated sweet corn cultivars. Crop Sci. 35:219-227.

Revilla P, P Soengas, R A Malvar, M E Cartea, A Ordás (1998) Isozyme variation and historical relationships among the maize races of Spain. Maydica 43:175-182.

Rohlf F J (1993) NTSYS-pc Numerical Taxonomy and Multivariate Analysis System. Version 1.8. Exeter Software. Setauket, NY, U.S.A.

Saitou N, M Nei (1987) The neighbor-joining method: a new method for reconstructing phylogenetic trees. Mol. Biol. Evol. 4:406-425.

Sánchez G J J, M M Goodman, C W Stuber (2000) Isozymatic and morphological diversity in the races of maize of Mexico. Econ. Bot. 54:43-59.

Santacruz-Varela A, M P Widrlechner, R J Salvador, K E Ziegler, M J Millard, P K Bretting (2004) Phylogenetic relationships among North American popcorns and their evolutionary links to Mexican and South American popcorns. Crop Sci. 44:14561467.

Smith J S C (1986) Genetic diversity whitin the corn belt dent racial complex of maize (Zea mays L.). Maydica 31:349-367.

SMN (2009) Productos Climatológicos. Servicio Meteorológico Nacional y Comisión Nacional del Agua. México. Disponible en: http://smn.cna.gob.mx/productos/productos.html (Febrero de 2009).

Snyder L A, D Freifelder, D L Hartl (1985) General Genetics. Jones and Bartlett. Boston, MA. $666 \mathrm{p}$.

Stuber C W, J F Wendel, M M Goodman, J S C Smith (1988) Techniques and scoring procedures for starch gel electrophoresis of enzymes from maize (Zea mays L.). North Carolina Agric. Res. Ser. Tech. Bull. 286. North Carolina State University. Raleigh, NC. $87 \mathrm{p}$

Wright S (1978) Evolution and the Genetics of Populations. Vol. 4. Variability Within and Among Natural Populations. University of Chicago Press. Chicago, IL. 628 p.

Yeh C F, R Yang, T Boyle (1999) POPGENE Version 1.31. Microsoft Windows-based Freeware for Population Genetic Analysis. Quik User Guide. University of Alberta and Center for International Forestry Research, Edmonton, AB. Canada. 29 p. 